\title{
Importancia Pericial de los Modelos de Yeso Odontológicos para la Identificación de Cuerpo Putrefacto - Relato de Caso
}

\author{
Forensic Importance of Dental Plaster Models for \\ Putrefied Body Identification - Case Report
}

\begin{abstract}
Rhonan Ferreira Silva'; Matheus Felter²; Pedro Henrique Moreira Paulo Tolentino²; Lívia Graziele Rodrigues²; Michelle Gouveia Benício de Araújo Andrade ${ }^{3}$; Alicia Mariel Picapedra Palomeque ${ }^{4}$ \& Carlos Sassi ${ }^{5}$
\end{abstract}

SILVA, R. F.; FELTER, M.; TOLENTINO, P. H. M. P.; RODRIGUES, L. G.; ANDRADE, M. G. B. A.; PALOMEQUE, A. M. P. \& SASSI, C. Importancia pericial de los modelos de yeso odontológicos para la identificación de cuerpo putrefacto - Relato de Caso. Int. J. Odontostomat., 11(3):273-278, 2017.

RESUMEN: Frente a casos complejos de identificación humana, como cuerpos en avanzado estado de descomposición, la aplicación de técnicas odontológicas se hace necesaria para establecer la identidad de una persona desaparecida. En este sentido, la existencia y obtención de registros odontológicos ante-mortem de una supuesta víctima, así como el análisis de las particularidades odontológicas del respectivo cadáver, puede constituirse en una posibilidad rápida y de bajo costo para la realización de pericias odontológicas de identificación humana. El objetivo de este relato es presentar un caso pericial, demostrando la importancia de los modelos de yeso para la identificación positiva de un cuerpo putrefacto, discutiendo los aspectos inherentes alarchivo y descarte de los mismos, después de su uso clínico. Los modelos de yeso odontológicos resultan sustanciales para el diagnóstico, planeamiento y ejecución de determinados tratamientos, debiendo ser almacenados en medio físico o digital. De igual manera, poseen relevante potencial para la identificación humana, al permitir, de forma confiable, la evaluación tridimensional de los dientes, sus posiciones en los arcos dentales y las peculiaridades odontológicas eventualmente presentes.

PALABRAS CLAVE: odontología legal, registros odontológicos, modelos dentales, prótesis dentales.

\section{INTRODUCCIÓN}

Con el aumento de la violencia urbana, el número de casos complejos de identificación humana, tales como carbonizados, esqueletizados, mutilados y putrefactos, viene aumentando cada año en los departamentos de medicina legal (Oliveira et al., 2015). En presencia de un cuerpo en estas condiciones, generalmente, no es posible hacer un análisis de las impresiones digitales para establecer la identidad de la víctima, siendo necesaria otra forma de identificación, echándose mano, habitualmente, al análisis de las particularidades cranianas (Prado et al., 2016) u odontológicas (Scoralick et al., 2013; Belotti et al., 2015; Silva et al., 2015a).
En este sentido, es posible verificar en la literatura pericial que los registros y particularidades odontológicas observadas en el examen ante-mortem (AM), de naturaleza anatómica, terapéutica o patológica, contenidas en fichas clínicas (Silva et al., 2015a), radiografías intra y/o extraorales (Scoralick et al.; Silva et al., 2016), fotografías (Terada et al., 2014), tomografías (Silva et al., 2011) y modelos de yeso (Silva et al., 2007), pueden ser adecuadamente utilizadas como subsidio para la identificación humana, desde que las mismas sean observadas en el examen post-mortem (PM), permitiendo así, una comparación odontológica.

\footnotetext{
${ }^{1}$ Odontología Legal, Facultad de Odontología de la Universidad Federal de Goiás. Perito Criminal de la Policía Científica de Goiás Goiânia, Goiás, Brasil.

${ }^{2}$ Facultad de Odontología de la Universidad Federal de Goiás, Goiânia, Goiás, Brasil.

${ }^{3}$ Odontología Legal de la Asociación Brasileña de Odontología, Goiânia, Goiás, Brasil.

${ }^{4}$ Servicio de Registro y Admisión de Pacientes de la Facultad de Odontología de la Universidad de la República, Montevideo, Uruguay.

${ }^{5}$ Doctorado en Anatomía de la Facultad de Odontología de Piracicaba de la Universidad Estadual de Campinas São Pablo, Brasil.
} 
SILVA, R. F.; FELTER, M.; TOLENTINO, P. H. M. P.; RODRIGUES, L. G.; ANDRADE, M. G. B. A.; PALOMEQUE, A. M. P. \& SASSI, C. Importancia pericial de los modelos de yeso odontológicos para la identificación de cuerpo putrefacto - Relato de Caso. Int. J. Odontostomat., 11(3):273-278, 2017.

Conscientes que la identificación humana, sirviéndose de modelos de yeso odontológicos no ha sido frecuentemente relatada en la literatura (Silva et al., 2007; Belotti et al.; Silva et al., 2015b), a pesar de que, en determinadas circunstancias puede ser el camino mas rápido para establecer la identidad de una persona desaparecida, el presente trabajo tiene como objetivo relatar un caso pericial, demostrando la importancia de los modelos de yeso para la identificación de un cuerpo putrefacto y discutir los aspectos inherentes al archivo y descarte de los mismos, después de su uso clínico.

\section{RELATO DE CASO}

Un cuerpo de individuo adulto o añoso, con características craneanas masculinas, fue encontrado en una residencia, en avanzada fase de descomposición, en un municipio del interior del estado de Goiás, región Centro-Oeste del Brasil. Luego del traslado del cadáver al Instituto Médico Legal (IML) correspondiente, la autopsia reveló que la muerte estaba asociada a causas naturales, no resultando posible el análisis de sus impresiones digitales.

En el examen odontológico PM se constató que la víctima era portadora de prótesis parciales removibles, dada su condición de desdentada anterosuperior y bilateral posteroinferior, Clase IV y I de Kennedy, respectivamente (Fig. 1). Como principales características odontológicas con potenciales identificadores, fueron observadas: la presencia de ter- ceros molares superiores erupcionados, restauraciones de amalgama, restauraciones de resina, pérdidas dentales antiguas en los espacios protéticos rehabilitados, apiñamientos, giroversiones y nichos para apoyo de prótesis removibles.

En un primer momento, fue colectado material para examen de ADN para la identificación de la víctima, siendo las muestras, cuestionada (del cadáver) y de referencia (de los familiares), enviadas a un laboratorio privado. Sin embargo, los resultados no fueron satisfactorios para establecer un vínculo genético entre los supuestos familiares, debido a que el material examinado estaba altamente degradado.

Por este motivo, se le solicitó a los familiares de la persona desaparecida (sexo masculino, 70 años de edad) que aportaran documentación odontológica para auxiliar en la identificación de la víctima, consistente en dos modelos de yeso, confeccionados tres años antes del óbito, para la realización de un juego de prótesis parciales removibles (Fig. 2). De este modo, fue posible verificar que estos correspondían a un individuo parcialmente desdentado, con la misma clasificación de Kennedy, particularidades odontológicas y dientes pilares para sustentación de las prótesis parciales removibles, que los observados en el examen PM. Asimismo, las prótesis parciales removibles, una vez posicionadas sobre los modelos de yeso, mostraron un correcto ajuste, como se ilustra en la Figura 3.

La comparación de las particularidades odontológicas encontradas en los modelos de yeso AM con las de los arcos dentales PM, arrojó 27 similitudes

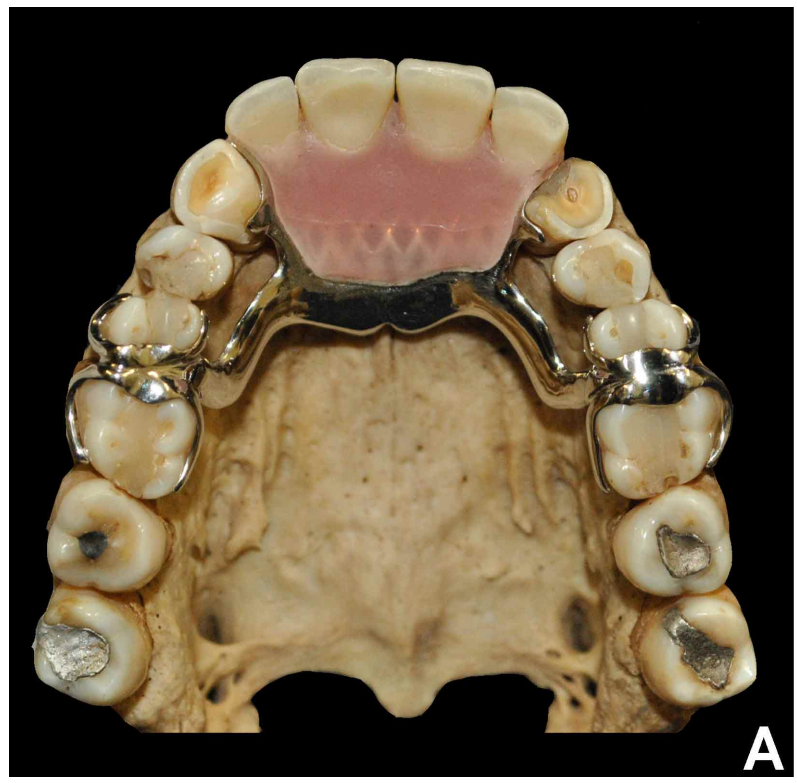

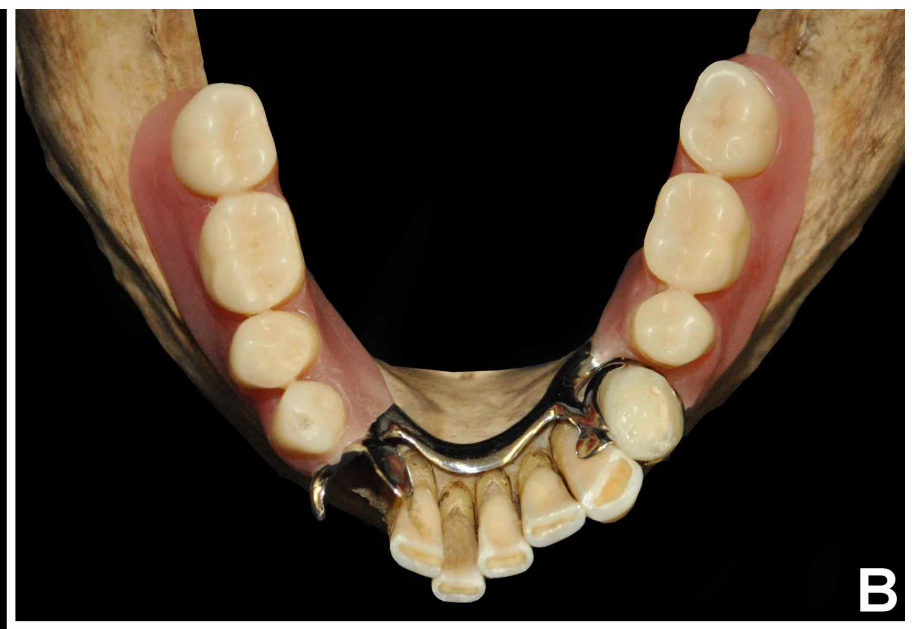

Fig. 1. Vista oclusal de los arcos dentales superior e inferior durante el examen PM. 
o coincidencias, 14 discrepancias explicables, no excluyentes, y ninguna discrepancia inexplicable, ex- cluyente, como se aprecia en las Tablas I y II, estableciéndose una identificación positiva de la víctima.

Tabla I. Comparación entre las particularidades odontológicas evidenciadas en los registros AM (modelo de yeso superior) y PM (cadáver) para el arco dental superior.

\begin{tabular}{|c|c|c|c|}
\hline Diente* & AM & PM & Comparación \\
\hline 11 & Pérdida antigua & Pérdida antigua & SI \\
\hline 12 & Pérdida antigua & Pérdida antigua & SI \\
\hline 13 & Corona íntegra & Corona íntegra & SI \\
\hline \multirow[t]{2}{*}{14} & Restauración** (OD) & Restauración de resina (OD) & DE \\
\hline & Giroversión & Giroversión & SI \\
\hline \multirow[t]{3}{*}{15} & Restauración** (MOD) & Restauración de resina (MOD) & DE \\
\hline & Giroversión & Giroversión & SI \\
\hline & Nicho protésico & Nicho protésico & SI \\
\hline \multirow[t]{2}{*}{16} & Restauración** (OD) & Restauración de resina (OD) & DE \\
\hline & Nicho protésico & Nicho protésico & SI \\
\hline 17 & Restauración** $(0)$ & Restauración de amalgama (O) & DE \\
\hline 18 & Restauración** (O) & Restauración de amalgama (O) & DE \\
\hline 21 & Pérdida antigua & Pérdida antigua & SI \\
\hline 22 & Pérdida antigua & Pérdida antigua & SI \\
\hline 23 & Corona integra & Corona fracturada & DE \\
\hline \multirow[t]{2}{*}{24} & Restauración** (OD) & Restauración de resina (OD) & $\mathrm{DE}$ \\
\hline & Giroversión & Giroversión & $\mathrm{SI}$ \\
\hline \multirow[t]{3}{*}{25} & Restauración** (MOD) & Restauración de resina (MOD) & DE \\
\hline & Giroversión & Giroversión & SI \\
\hline & Nicho protésico & Nicho protésico & SI \\
\hline \multirow[t]{2}{*}{26} & Restauración** (MO) & Restauración de resina (MO) & DE \\
\hline & Nicho protésico & Nicho protésico & SI \\
\hline 27 & Restauración** $(\mathrm{O})$ & Restauración de amalgama (O) & DE \\
\hline 28 & Restauración** (O) & Restauración de amalgama (O) & DE \\
\hline
\end{tabular}

SI: Similitud; DE: Discrepancia Explicable. *Notación dental preconizada por la FDI. **Material restaurador no puede ser identificado.

Tabla II. Comparación entre las particularidades odontológicas evidenciadas en los registros AM (modelo de yeso inferior) y PM (cadáver) para el arco dental inferior.

\begin{tabular}{cllc}
\hline Diente* $^{*}$ & AM & PM & Comparación \\
\hline 31 & Desgaste incisal & Desgaste incisal & $\mathrm{SI}$ \\
32 & Desgaste incisal & Desgaste incisal & $\mathrm{SI}$ \\
33 & Desgaste incisal & Desgaste incisal & $\mathrm{SI}$ \\
34 & Corona irregular** & Corona de resina composta & $\mathrm{DE}$ \\
35 & Pérdida antigua & Pérdida antigua & $\mathrm{SI}$ \\
36 & Pérdida antigua & Pérdida antigua & $\mathrm{SI}$ \\
37 & Pérdida antigua & Pérdida antigua & $\mathrm{SI}$ \\
38 & Pérdida antigua & Pérdida antigua & $\mathrm{SI}$ \\
41 & Borde Incisal fracturado & Desgaste incisal & $\mathrm{DE}$ \\
& Vestibularizado & Vestibularizado & $\mathrm{SI}$ \\
42 & Desgaste incisal & Desgaste incisal & $\mathrm{SI}$ \\
43 & Desgaste incisal & Pérdida post-mortem & $\mathrm{DE}$ \\
44 & Pérdida antigua & Pérdida antigua & $\mathrm{SI}$ \\
45 & Pérdida antigua & Pérdida antigua & $\mathrm{SI}$ \\
46 & Pérdida antigua & Pérdida antigua & $\mathrm{SI}$ \\
47 & Pérdida antigua & Pérdida antigua & $\mathrm{SI}$ \\
48 & Pérdida antigua & Pérdida antigua & $\mathrm{SI}$ \\
\hline
\end{tabular}

SI: Similitud; DE: Discrepancia Explicable. *Notación dental preconizada por la FDI. **Material restaurador no puede ser identificado. 


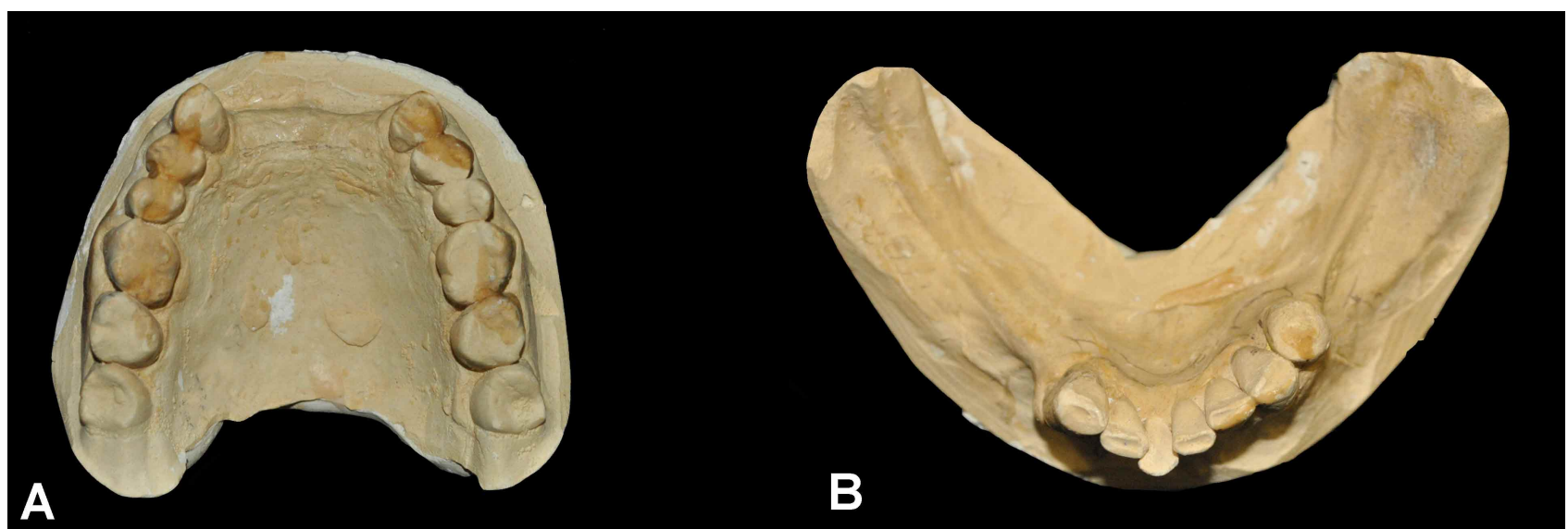

Fig. 2. Vista oclusal de los modelos de yeso superior e inferior AM.

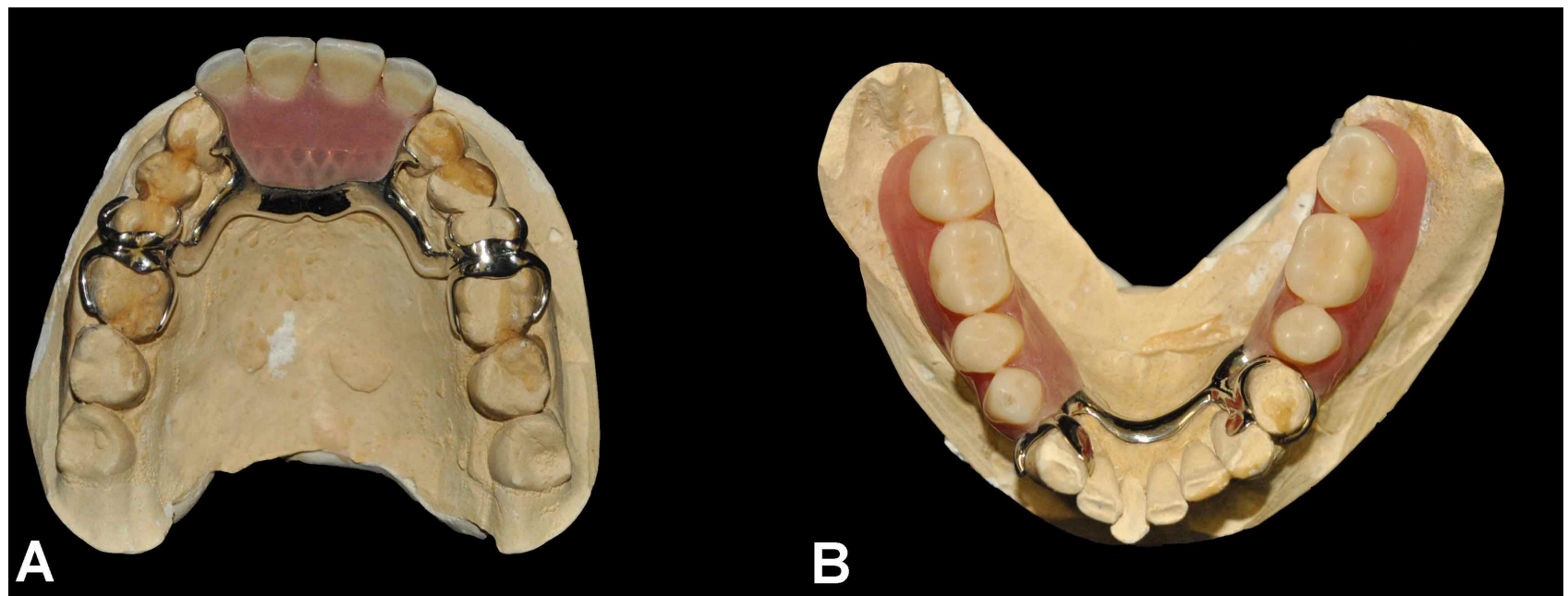

Fig. 3. Prótesis removibles que fueran encontradas en los arcos dentales del cadáver, posicionadas sobre los modelos de yeso AM.

\section{DISCUSIÓN}

La identificación humana en casos complejos, como los de cuerpos carbonizados, esqueletizados y putrefactos, generalmente se basa en las particularidades odontológicas, pues en estas circunstancias, los tejidos blandos que componen las impresiones digitales poseen baja resistencia, tanto a la acción del calor/fuego (Recalde et al., 2015) como a la intemperie y al proceso de descomposición, respecto a los dientes y materiales odontológicos (Scoralick et al.; Belotti et al.; Silva et al., 2015a).

El método odontológico obtiene resultados rápidos y de bajo costo si se los compara a los de los exámenes de ADN. Por otra parte, cada vez más población tiene acceso a tratamientos odontológicos, ya sea de rutina o especializados, lo que aumenta la cantidad de documentos con potencial o finalidad pericial, como fichas clínicas, radiografías, tomografías, fotografías y modelos de yeso (Scoralick et al.; Belotti et al.; Silva et al., 2015a).

Desde el punto de vista operacional, cada tipo de registro de la documentación odontológica posee ventajas y desventajas para el proceso de identificación humana. Las fichas e historias clínicas permiten que el odontólogo, aún sujeto a fallas humanas que puedan comprometer el resultado final, asiente los procedimientos ejecutados en un tratamiento, explicitando caras, dientes y materiales empleados (Silva et al., 2015a; Silva et al., 2015c). A su vez, las radiografías 
facilitan la evaluación cuali y cuantitativa de las particularidades odontológicas (anatómicas, terapéuticas y patológicas), pero con la limitación de ser bidimensionales, produciéndose por tanto, una superposición de imágenes propia del examen realizado (Scoralick et al.; Silva et al., 2016). Las tomografías permiten un análisis más detallado de una determinada región, teniendo como principal inconveniente su alto costo, por lo cual sus indicaciones y uso se ven restringidos en la práctica rutinaria de los IMLs (Silva et al., 2011). Finalmente, los modelos de yeso, aunque no sean confeccionados en todos los tipos de terapia, se tornan necesarios para el plan y ejecución de tratamientos ortodóncicos y protésicos, y de utilidad pericial, al facultar el análisis tridimensional de los dientes y estructuras reproducidas (Silva et al., 2007; Belotti et al.; Silva et al., 2015b), así como el de las rugosidades palatinas (Argollo et al., 2017), para su comparación con el material PM cuando estén preservadas, lo que no sucedió en el caso en cuestión, por el avanzado estado de descomposición cadavérica.

En el presente relato, fue entregado para la pericia, un juego de modelos de yeso realizado con finalidad protésica, lo que permitió el análisis de la morfología y posición de los dientes remanentes, al igual que la constatación de restauraciones odontológicas y nichos para el apoyo de las prótesis parciales removibles encontradas en el cadáver. Cabe destacar, que no fue posible la identificación del material restaurador presente en los dientes remanentes, pero sí verificar las características superficiales de las preparaciones cavitarias, tales como su extensión y límites en fosas, cúspides y crestas marginales, e inclusive, eventuales desadaptaciones (Silva et al., 2015b).

En relación a los puntos considerados en la comparación odontológica, se obtuvieron 27 similitudes y 14 discrepancias explicables, arribándose a una identificación positiva, resaltándose que, este resultado no depende de un número mínimo de puntos (Acharya \& Taylor, 2003; Belotti et al.), por no tratarse de una evaluación simplemente cuantitativa, a diferencia de la papiloscópica.

Considerando el potencial forense de los modelos de yeso para la identificación humana, adquiere trascendencia que estas reproducciones sean archivadas, por el mayor tiempo posible, junto a la historia clínica odontológica de los pacientes (Silva et al., 2007). En caso de una hipotética falta de espacio, sería aconsejable digitalizar/fotografiar los modelos para que sus imágenes formasen parte de un banco digital de informaciones de los pacientes, como es recomendado en algunos países sudamericanos (Conselho Federal de Odontologia, 2012; Uruguay, 2005), el cual podría ser introducido en sistemas electrónicos de gerenciamiento de consultorios, liberando la entrega de los modelos físicos a los pacientes, mediante firma del concerniente recibo o de la constancia del hecho en su historia clínica.

En suma, los modelos de yeso odontológicos, además de desempeñar un significativo rol en el diagnóstico, planeamiento y ejecución de ciertos tratamientos, poseen relevante potencial para la identificación humana, al posibilitar la evaluación tridimensional de los dientes, sus posiciones en los arcos dentales y las peculiaridades odontológicas eventualmente presentes, de forma rápida, confiable y a costos reducidos.

SILVA, R. F.; FELTER, M.; TOLENTINO, P. H. M. P.; RODRIGUES, L. G.; ANDRADE, M. G. B. A.; PALOMEQUE, A. M. P. \& SASSI, C. Forensic importance of dental plaster models for putrefied body identification - Case Report. Int. J. Odontostomat., 11(3):273-278, 2017.

ABSTRACT: Faced with complex cases of human identification, such as bodies in an advanced state of decomposition, the application of dental techniques becomes necessary to establish the identity of a missing person. In this sense, the existence and obtaining of ante-mortem dental records of an alleged victim, as well as the analysis of the dental specificities of the respective corpse, can constitute a quick and low-cost possibility for human identification by dental features. The objective of this report is to present a forensic case, demonstrating the importance of plaster models for the positive identification of a decomposed body, discussing the inherent aspects of archiving and discarding them after their clinical use. Dental plaster models are substantial for the diagnosis, planning and execution of certain treatments, and must be stored on physical or digital media. Likewise, they have a significant potential for human identification by reliably allowing the three-dimensional evaluation of the teeth, their positions in the dental arches and the dental peculiarities that may be present.

KEY WORDS: forensic dentistry, dental records, dental models, dental prosthesis.

\section{REFERENCIAS BIBLIOGRÁFICAS}

Acharya, A. B. \& Taylor, J. A. Are a minimum number of concordant matches needed to establish identity in forensic odontology? $\mathrm{J}$. Forensic Odontostomatol., 21(1):6-13, 2003.

Argollo, S. P.; Argollo, B. P.; Argollo, P. A. N. \& Marques, J. A. M. 
SILVA, R. F.; FELTER, M.; TOLENTINO, P. H. M. P.; RODRIGUES, L. G.; ANDRADE, M. G. B. A.; PALOMEQUE, A. M. P. \& SASSI, C. Importancia pericial de los modelos de yeso odontológicos para la identificación de cuerpo putrefacto - Relato de Caso. Int. J. Odontostomat., 11(3):273-278, 2017.

Utilização da rugoscopia palatina para identificação de corpo carbonizado - Relato de caso pericial. R. B. O. L. Rev. Bras. Odontol. Leg., 4(1):107-13, 2017.

Belotti, L.; Rabbi, R.; Pereira, S. D. R.; Barbosa, R. S.; Carvalho, K. S. \& Pacheco, K. T. S. É possível identificar positivamente um corpo carbonizado somente por dois dentes? Relato de um caso pericial. R. B. O. L. Rev. Bras. Odontol. Leg., 2(2):105-15, 2015.

Conselho Federal de Odontologia. Resolução CFO 118 de 11 de maio de 2012. Revoga o Código de Ética Odontológica aprovado pela Resolução CFO-42/2003 e aprova outro em substituição. Rio de Janeiro, Conselho Federal de Odontologia, 2012. Disponible en: http://cfo.org.br/wp-content/uploads/2009/09/ codigo_etica.pdf

Oliveira, M. C. A.; Vieira, E. L. R.; Penha, M. R. C.; Melo, E. H. \& Caldas Junior, A. F. Characterization of women victims of violent death in a metropolitan area of Northeast Brazil. R. G. O. Rev. Gaúch. Odontol., 63(4):439-45, 2015.

Prado, F. B.; Freire, A. R.; Rondon, B. C. S.; Costa, S. T.; Rossi, A. C. \& Daruge Júnior, E. Analysis of the frontal sinus morphology and the titanium plates shape in skull fracture for human identification. Int. J. Odontostomatol., 10(2):303-8, 2016.

Recalde, T. F.; Dias, P. E. M.; Rodríguez, J. C. Z.; Escobar, M. C. G. \& Perdomo, M. C. Análisis del comportamiento de los tejidos dentales y materiales de obturación endodóncicos sometidos a altas temperaturas con fines forenses. R. B. O. L. Rev. Bras. Odontol. Leg., 2(1):35-45, 2015.

Scoralick, R. A.; Barbieri, A. M.; Moraes, Z. M.; Francesquini Júnior, L.; Daruge Júnior, E. \& Naressi, S. C. M. Human identification through dental radiographs study: case report. Rev. Odontol. U. N. E.S. P., 42(1):67-71, 2013.

Silva, R. F.; Botelho, T. L.; Prado, F. B.; Kawagushi, J. T.; Daruge Júnior, E. \& Bérzin, F. Human identification based on cranial computed tomography scan: a case report. Dentomaxillofac. Radiol., 40(4):257-61, 2011.

Silva, R. F.; Dias, P. E. M.; Picoli, F. F.; Rodrigues, L. G.; Mundim, M. B. V. \& Franco, A. Inconsistências antropológicas observadas em corpo putrefeito identificado por registros odontológicos $R$ relato de caso pericial. R. B. O. L. Rev. Bras. Odontol. Leg., 2(1):125-36, 2015a

Silva, R. F.; Franco, A.; Picoli, F. F.; Rodrigues, L. G. \& Resende, R. G. Dental human identification using bitewing radiographs $-A$ case report. Eur. J. Forensic Sci., 3(3):31-3, 2016.

Silva, R. F.; Mundim, M. B. V.; Picoli, F. F. \& Franco, A. Dental identification of a mummified body using dental cast and prosthesis. J. Forensic Investig., 3(2):3, 2015b.

Silva, R. F.; Oliveira, C. F. P.; Souza, P. O. C.; Rodrigues, L. G.; Mundim, M. B. V.; Franco, A. \& Picoli, F. F. Repercussões periciais diante de falha no preenchimento de prontuário odontológico Relato de caso pericial. Braz. J. Forensic Sci. Med. Law Bioeth., 4(2):209-17, 2015c.

Silva, R. F.; Ramos, D. I. A.; Pereira, S. D. R.; Daruge, E. \& Daruge Júnior, E. Model cast: expertise relevance and forensic orientation for filing away. Rev. Assoc. Paul. Cir. Dent., 61(5):381-4, 2007.

Terada, A. S. S. D.; Silva, R. H. A.; Guimarães, M. A.; Silveira, T. C. P.; Paranhos, L. R. \& Araujo, L. G. Orthodontic use of documentation in identification of a skeletonized body in legal dental practice. Int. J. Odontostomatol., 8(1):41-6, 2014.

Uruguay. Decreto No $37 / 005$ de 27 de enero de 2005. Regula la Destrucción de Historias Clínicas y la Aplicación de Tecnología Informática. Modifica en los Artículos 4 y 11 el Decreto 355/982. Red Iberoamericana de Protección de Datos, 2005. Disponible en: http://www.redipd.org/legislacion/common/legislacion/ uruguay/decreto-37-005.pdf.
Dirección para correspondencia:

Rhonan Ferreira da Silva

Instituto Medico-legal

Avenida Atilio Correa Lima, 1223

Cidade Jardim

Goiânia, Goiás 74425-030

BRASIL

E-mail: rhonansilva@gmail.com

Recibido : 14-04-2017

Aceptado: 12-06-2017 